\title{
On the Chromatic Number of Disjointness Graphs of Curves
}

\author{
János Pach \\ École Polytechnique Fédérale de Lausanne, Lausanne, Switzerland \\ Rényi Institute, Budapest, Hungary \\ janos.pach@epfl.ch
}

\section{István Tomon}

École Polytechnique Fédérale de Lausanne, Lausanne, Switzerland istvan.tomon@epfl.ch

\begin{abstract}
Let $\omega(G)$ and $\chi(G)$ denote the clique number and chromatic number of a graph $G$, respectively. The disjointness graph of a family of curves (continuous arcs in the plane) is the graph whose vertices correspond to the curves and in which two vertices are joined by an edge if and only if the corresponding curves are disjoint. A curve is called $x$-monotone if every vertical line intersects it in at most one point. An $x$-monotone curve is grounded if its left endpoint lies on the $y$-axis.

We prove that if $G$ is the disjointness graph of a family of grounded $x$-monotone curves such that $\omega(G)=k$, then $\chi(G) \leq\left(\begin{array}{c}k+1 \\ 2\end{array}\right)$. If we only require that every curve is $x$-monotone and intersects the $y$-axis, then we have $\chi(G) \leq \frac{k+1}{2}\left(\begin{array}{c}k+2 \\ 3\end{array}\right)$. Both of these bounds are best possible. The construction showing the tightness of the last result settles a 25 years old problem: it yields that there exist $K_{k}$-free disjointness graphs of $x$-monotone curves such that any proper coloring of them uses at least $\Omega\left(k^{4}\right)$ colors. This matches the upper bound up to a constant factor.
\end{abstract}

2012 ACM Subject Classification Mathematics of computing $\rightarrow$ Graph theory

Keywords and phrases string graph, chromatic number, intersection graph

Digital Object Identifier 10.4230/LIPIcs.SoCG.2019.54

Related Version https://arxiv.org/abs/1811.09158

Funding János Pach: Research partially supported by Swiss National Science Foundation grants no. 200020-162884 and 200021-175977.

István Tomon: Research partially supported by Swiss National Science Foundation grants no. 200020-162884 and 200021-175977.

Acknowledgements We would like to thank Andrew Suk, Gábor Tardos, Géza Tóth and Bartosz Walczak for fruitful discussions.

\section{Introduction}

Given a family of sets, $\mathcal{C}$, the intersection graph of $\mathcal{C}$ is the graph, whose vertices correspond to the elements of $\mathcal{C}$, and two vertices are joined by an edge if the corresponding sets have a nonempty intersection. Also, the disjointness graph of $\mathcal{C}$ is the complement of the intersection graph of $\mathcal{C}$, that is, two vertices are joined by an edge if the corresponding sets are disjoint. As usual, we denote the clique number, the independence number, and the chromatic number of a graph $G$ by $\omega(G), \alpha(G)$ and $\chi(G)$, respectively. 


\subsection{Clique number vs. chromatic number}

Computing these parameters for intersection graphs of various classes of geometric objects (segments, boxes, disks etc.) or for other geometrically defined graphs (such as visibility graphs) is a computationally hard problem and a classic topic in computational and combinatorial geometry $[1,5,10,17,23,24]$. There are many interesting results connecting the clique number and the chromatic number of geometric intersection graphs, starting with a beautiful theorem of Asplund and Grünbaum [2], which states that every intersection graph $G$ of axis-parallel rectangles in the plane satisfies $\chi(G) \leq 4(\omega(G))^{2}$.

A family $\mathcal{G}$ of graphs is $\chi$-bounded if there exists a function $f: \mathbb{Z}^{+} \rightarrow \mathbb{Z}^{+}$such that every $G \in \mathcal{G}$ satisfies $\chi(G) \leq f(\omega(G))$. In this case, say that the function $f$ is $\chi$-bounding for $\mathcal{G}$. Using this terminology, the result of Asplund and Grünbaum [2] mentioned above can be rephrased as follows: The family of intersection graphs of axis-parallel rectangles in the plane is $\chi$-bounded with bounding function $f(k)=4 k^{2}$. (It is conjectured that the same is true with bounding function $f(k)=O(k)$.) However, an ingenious construction of Burling [4] shows that the family of intersection graphs of axis-parallel boxes in $\mathbb{R}^{3}$ is not $\chi$-bounded. The $\chi$-boundedness of intersection graphs of chords of a circle was established by Gyárfás [14, 15]; see also Kostochka et al. [22, 19, 20].

Computing the chromatic number of the disjointness graph of a family of objects, $\mathcal{C}$, is equivalent to determining the clique cover number of the corresponding intersection graph $G$, that is, the minimum number of cliques whose vertices together cover the vertex set of $G$. This problem can be solved in polynomial time only for some very special families (for instance, if $\mathcal{C}$ consists of intervals along a line or arcs along a circle [13]). On the other hand, the problem is known to be NP-complete if $\mathcal{C}$ is a family of chords of a circle $[16,12]$ or a family of unit disks in the plane $[38,6]$, and in many other cases. There is a vast literature providing approximation algorithms or inapproximability results for the clique cover number $[8,9]$.

\subsection{Families of curves}

A curve or string in $\mathbb{R}^{2}$ is the image of a continuous function $\phi:[0,1] \rightarrow \mathbb{R}^{d}$. A curve $C \subset \mathbb{R}^{2}$ is called $x$-monotone if every vertical line intersects $C$ in at most one point. Note that any convex set can be approximated arbitrarily closely by $x$-monotone curves, so the notion of $x$-monotone curve extends the notion of convex sets. We say that $C$ is grounded at the curve $L$ if one of the endpoints of $C$ is in $L$, and this is the only intersection point of $C$ and $L$. A grounded $x$-monotone curve is an $x$-monotone curve that is contained in the half-plane $\{x \geq 0\}$, and whose left endpoint lies on the vertical line $\{x=0\}$.

It was first suggested by Erdős in the 1970s, and remained the prevailing conjecture for 40 years, that the family of intersection graphs of curves (the family of so-called "string graphs") is $\chi$-bounded $[3,21]$. There were many promising facts pointing in this direction. Extending earlier results of McGuinness [28], Suk [37], and Lasoń et al. [27], Rok and Walczak [35, 36] proved the conjecture for grounded families of curves. Nevertheless, in 2014, Pawlik et al. [34] disproved Erdős's conjecture. They managed to modify Burling's above-mentioned construction to obtain a sequence of finite families of segments in the plane whose intersection graphs, $G_{n}$, are triangle-free (that is, $\omega\left(G_{n}\right)=2$ ), but their chromatic numbers tend to infinity, as $n \rightarrow \infty$.

Recently, Pach, Tardos and Tóth [32] proved that the family of disjointness graphs of curves in the plane is not $\chi$-bounded either; see also [30]. However, the situation is different if we restrict our attention to $x$-monotone curves. It was shown in $[33,26]$ that the family 
of disjointness graphs of $x$-monotone curves in the plane is $\chi$-bounded with a bounding function $f(k)=k^{4}$. For grounded $x$-monotone curves, the same proof provides a better bounding function: $f(k)=k^{2}$. These results proved 25 years ago were not likely to be tight. However, in spite of many efforts, no-one has managed to improve them or to show that they are optimal.

\subsection{Our results}

The aim of the present paper is to fill this gap. We proved, much to our surprise, that the order of magnitude of the last two bounds cannot be improved. In fact, in the case of grounded $x$-monotone curves, we determined the exact value of the best bounding function for every $k \geq 2$. To the best of our knowledge, this is the first large family of non-perfect geometric disjointness graphs, for which one can precisely determine the best bounding function.

- Theorem 1. Let $G$ be the disjointness graph of a family of grounded $x$-monotone curves. If $\omega(G)=k$, then $\chi(G) \leq\left(\begin{array}{c}k+1 \\ 2\end{array}\right)$.

Theorem 2. For every positive integer $k \geq 2$, there exists a family $\mathcal{C}$ of grounded $x$ monotone curves such that if $G$ is the disjointness graph of $\mathcal{C}$, then $\omega(G)=k$ and $\chi(G)=$ $\left(\begin{array}{c}k+1 \\ 2\end{array}\right)$.

It turns out that disjointness graphs of grounded $x$-monotone curves can be characterized by two total orders defined on their vertex sets that satisfy some special properties. This observation is the key idea behind the proof of the above two theorems.

The disjointness graph of any collection of $x$-monotone curves, each of which intersects a given vertical line (the $y$-axis, say), is the intersection of two disjointness graphs of grounded $x$-monotone curves. The methods used for proving Theorems 1 and 2 can be extended to such disjointness graphs and yield sharp bounds.

- Theorem 3. Let $G$ be the disjointness graph of a family $\mathcal{C}$ of $x$-monotone curves such that all elements of $\mathcal{C}$ have nonempty intersection with a vertical line $l$. If $\omega(G)=k$, then $\chi(G) \leq \frac{k+1}{2}\left(\begin{array}{c}k+2 \\ 3\end{array}\right)$.

- Theorem 4. For every positive integer $k \geq 2$, there exists a family $\mathcal{C}$ of $x$-monotone curves such that all elements of $\mathcal{C}$ have nonempty intersection with a vertical line $l$, the disjointness graph $G$ of $\mathcal{C}$ satisfies $\omega(G)=k$, and $\chi(G)=\frac{k+1}{2}\left(\begin{array}{c}k+2 \\ 3\end{array}\right)$.

As we have mentioned before, according to [33, 26], $k^{4}$ is a bounding function for disjointness graphs of any family of $x$-monotone curves. Theorem 4 implies that the order of magnitude of this bounding function is best possible. Actually, we can obtain a little more.

- Theorem 5. For any positive integer $k$, let $f(k)$ denote the smallest $m$ such that any $K_{k+1}$-free disjointness graph of $x$-monotone curves can be properly colored with $m$ colors. Then we have

$$
\frac{k+1}{2}\left(\begin{array}{c}
k+2 \\
3
\end{array}\right) \leq f(k) \leq k^{2}\left(\begin{array}{c}
k+1 \\
2
\end{array}\right) .
$$

Here the lower and upper bounds differ by a factor of less than 6 , and there is some hope that one can determine the exact value of $f(k)$. The lower bound follows directly from Theorem 4.

Our paper is organized as follows. In Section 2, we prove Theorem 1 and the upper bound in Theorem 5. The existence of the graphs satisfying Theorem 2 is proved in Section 3, using probabilistic techniques. The proofs of Theorems 3 and 4 are presented in Sections 4 and 5, respectively. The last section contains open problems and concluding remarks. 


\section{A bounding function for grounded curves - Proofs of Theorems 1 and 5}

First, we establish Theorem 1. As usual, we denote the set $\{1,2, \ldots, n\}$ by $[n]$.

An ordered graph $G_{<}$is a graph, whose vertex set is endowed with a total ordering <. Ordered graphs are often more suitable for modelling geometric configurations than unordered ones; see, e.g., $[11,31]$. To model families of grounded $x$-monotone curves, we introduce a class of ordered graphs.

- Definition 6. An ordered graph $G_{<}$is called a semi-comparability graph, if it has no 4 vertices $a, b, c, d \in V\left(G_{<}\right)$such that $a<b<c<d$ and $a b, b c, c d \in E\left(G_{<}\right)$, but $a c, b d \notin E\left(G_{<}\right)$.

An unordered graph $G$ is said to be a semi-comparability graph, if its vertex set has a total ordering $<$ such that $G_{<}$is a semi-comparability graph.

Obviously, every comparability graph (that is, every graph whose edge set consists of all comparable pairs of a partially ordered set) is a semi-comparability graph.

- Lemma 7. The disjointness graph of every family $\mathcal{C}$ of grounded $x$-monotone curves is a semi-comparability graph.

Proof. Let $G$ be the disjointness graph of $\mathcal{C}$. Identify the vertices of $G$ with the elements of $\mathcal{C}$. For any $\gamma \in \mathcal{C}$, let $\left(0, y_{\gamma}\right)$ be the left endpoint of $\gamma$. Slightly perturbing the curves if necessary, we can assume without loss of generality that no two $y_{\gamma}$ s coincide. Let $<$ be the total ordering on $V(G)$, according to which $\gamma<\gamma^{\prime}$ if and only if $y_{\gamma}<y_{\gamma^{\prime}}$.

Suppose for contradiction that there exist 4 curves $a, b, c, d$ such that $a<b<c<d$ and $a b, b c, c d \in E(G)$, but $a c, b d \notin E(G)$. Then $a$ and $c$ must intersect, which means that $a, c$, and the ground line $x=0$ enclose a region $A$. Since $b$ does not intersect either of $a$ or $c$, it must lie in $A$. In order to intersect $b, d$ has to cross $c$, which is a contradiction.

By the dual of Dilworth's theorem [7], also known as Mirsky's theorem [29], comparability graphs are perfect. Thus, any comparability graph $G$ can be properly colored with $\omega(G)$ colors. While not all semi-comparability graphs are perfect, they are $\chi$-bounded.

- Lemma 8. For any semi-comparability graph $G$ with $\omega(G)=k$, we have $\chi(G) \leq\left(\begin{array}{c}k+1 \\ 2\end{array}\right)$.

Proof. Fix an ordering $<$ of $V(G)$ such that $G_{<}$is a semi-comparability graph. For every $v \in V(G)$, let $f(v)$ denote the size of the largest clique with minimal element $v$. Then $f(v) \in[k]$. For $i=1, \ldots, k$, let $V_{i}=\{v \in G: f(v)=i\}$.

The main observation is that $G\left[V_{i}\right]$ is a partial order. Indeed, suppose to the contrary that there exist 3 vertices $a, b, c \in V_{i}$ such that $a<b<c$ and $a b, b c \in E(G)$, but $a c \notin E(G)$. Let $C \subset V(G)$ be a clique of size $i$ with minimal element $c$. If $d \in C \backslash\{c\}$, then $b$ and $d$ must be joined by an edge, otherwise the quadruple $a, b, c, d$ satisfies the conditions $a b, b c, c d \in E(G)$ and $a c, b d \notin E(G)$. Thus, $b$ is joined to every vertex in $C$ by an edge, which means that $C \cup\{b\}$ is a clique of size $i+1$ with minimal element $b$, contradicting our assumption that $b \in V_{i}$.

Hence, every $G\left[V_{i}\right]$ is a partial order. Using the fact that $G\left[V_{i}\right]$ does not contain a clique of size $i+1$, by Mirsky's theorem [7] we obtain that $\chi\left(G\left[V_{i}\right]\right) \leq i$. Summing up for all $i$, we get that $\chi(G) \leq \sum_{i=1}^{k} \chi\left(G\left[V_{i}\right]\right) \leq\left(\begin{array}{c}k+1 \\ 2\end{array}\right)$, as required.

The combination of Lemmas 7 and 8 immediately implies Theorem 1 .

Next, we prove the upper bound in Theorem 5 . 
- Theorem 9. Let $G$ be the disjointness graph of a collection of $x$-monotone curves with $\omega(G)=k$. Then we have $\chi(G) \leq k^{2}\left(\begin{array}{c}k+1 \\ 2\end{array}\right)$.

Proof. Let $\mathcal{C}$ be a collection of $x$-monotone curves satisfying the conditions in the theorem. For any $\gamma \in \mathcal{C}$, let $x(\gamma)$ denote the projection of $\gamma$ to the $x$-axis. For $\alpha, \beta \in \mathcal{C}$, let $\alpha \prec \beta$ if $\min x(\alpha)<\min x(\beta)$ and $\max x(\alpha)<\max x(\beta)$.

Suppose that $\alpha$ and $\beta$ are disjoint. Let $\alpha<_{1} \beta$ if $\alpha \prec \beta$ and $\alpha$ is below $\beta$, that is, if on every vertical line that intersects both $\alpha$ and $\beta$, the intersection point of $\alpha$ lies below the intersection point of $\beta$. Let $\alpha<_{2} \beta$ if $\alpha \prec \beta$ and $\beta$ is below $\alpha$. Clearly, $<_{1}$ and $<_{2}$ are partial orders.

As $\omega(G) \leq k$, the size of the longest chains with respect to $<_{1}$ and $<_{2}$ is at most $k$. Therefore, the vertices of $G$ can be colored with $k^{2}$ colors such that each color class is an antichain in both $<_{1}$ and $<_{2}$.

It remains to show that each of these color classes can be properly colored with $\left(\begin{array}{c}k+1 \\ 2\end{array}\right)$ colors. Let $\mathcal{C}^{\prime} \subset \mathcal{C}$ such that no two elements of $\mathcal{C}^{\prime}$ are comparable by $<_{1}$ or $<_{2}$. Then, if $\alpha, \beta \in \mathcal{C}^{\prime}$, then either $\alpha$ and $\beta$ intersect, or one of the intervals $x(\alpha)$ or $x(\beta)$ contains the other. In either case, $x(\alpha)$ and $x(\beta)$ have a nonempty intersection, so any two elements of $\left\{x(\gamma): \gamma \in \mathcal{C}^{\prime}\right\}$ intersect. Hence, $\bigcap_{\gamma \in \mathcal{C}^{\prime}} x(\gamma)$ is nonempty, and there exists a vertical line $l$ that intersects every element of $\mathcal{C}^{\prime}$.

Let $G^{\prime}$ denote the disjointness graph of $\mathcal{C}^{\prime}$. Order the elements of $\mathcal{C}^{\prime}$ with respect to their intersections with $l$, from bottom to top. We claim that the resulting ordered graph $G_{<}^{\prime}$ is a semi-comparability graph. Indeed, suppose to the contrary that there are four vertices $a, b, c, d \in V\left(G^{\prime}\right)$ such that $a<b<c<d$ and $a b, b c, c d \in E\left(G^{\prime}\right)$, but $a c, b d \notin E\left(G^{\prime}\right)$. Without loss of generality, suppose that the length of $x(b)$ is larger than the length of $x(c)$; the other case can be handled similarly. As $b c \in E\left(G^{\prime}\right)$, we have $x(c) \subset x(b)$ and $b$ is below $c$, so every vertical line intersecting $c$ intersects $b$ as well, and its intersection with $b$ lies below its intersection with $c$. Also, as $a b \in E\left(G^{\prime}\right)$, we have that $a$ is below $b$. But then $a$ and $c$ must be disjoint, contradicting the condition $a c \notin E\left(G^{\prime}\right)$.

Thus, we can apply Lemma 8 to conclude that $G^{\prime}$ can be properly colored with $\left(\begin{array}{c}k+1 \\ 2\end{array}\right)$ colors. This completes the proof.

Let $g(n)$ denote the maximal number $m$ such that every collection of $n$ convex sets in the plane contains $m$ elements that are either pairwise disjoint, or pairwise intersecting. Larman et al. [26] proved that $g(n) \geq n^{1 / 5}$, while the best known upper bound, due to Kynčl [25] is $g(n)<n^{\log 8 / \log 169} \approx n^{0.405}$. Theorem 9 implies the following modest improvement on the lower bound.

- Corollary 10. Every collection of $n$-monotone curves (or convex sets) in the plane contains $((2+o(1)) n)^{1 / 5} \approx 1.15 n^{1 / 5}$ elements that are either pairwise disjoint or pairwise intersecting.

Proof. In every graph $G$ on $n$ vertices, we have $\alpha(G) \chi(G) \geq n$. In view of Theorem 9 , this implies that if $\mathcal{C}$ is a collection of $n x$-monotone curves and $G$ is the disjointness graph of $\mathcal{C}$, then we have

$$
\alpha(G)(\omega(G))^{3} \frac{\omega(G)+1}{2} \geq n .
$$

Therefore, $\max \{\alpha(G), \omega(G)\} \geq((2+o(1)) n)^{1 / 5}$, as claimed.

Many attempts were made to improve the order of magnitude of the lower bound on $g(n)$. It appeared to be conceivable to cover the disjointness graph $G$ of any collection of $x$-monotone curves with fewer than 4 comparability graphs, which would have yielded $\chi(G) \leq(\omega(G))^{3}$ and $g(n) \geq n^{1 / 4}$. These hopes are shattered by Theorem 4 . 


\section{Magical graphs - Proof of Theorem 2}

The converse of Lemma 7 is not true: not every semi-comparability graph can be realized as the disjointness graph of a collection of grounded $x$-monotone curves. See Section 6 , for further discussion. To characterize such disjointness graphs, we need to introduce a new family of graphs.

A graph $G_{<_{1},<_{2}}$ with two total orderings, $<_{1}$ and $<_{2}$, on its vertex set is called doubleordered. If the orderings $<_{1},<_{2}$ are clear from the context, we shall write $G$ instead of $G_{<_{1},<_{2}}$.

- Definition 11. A double-ordered graph $G_{<_{1},<_{2}}$ is called magical if for any three distinct vertices $a, b, c \in V(G)$ with $a<_{1} b<_{1} c$, if $a b, b c \in E(G)$ and $a c \notin E(G)$, then $b<_{2}$ a and $b<_{2} c$.

A graph $G$ is said to be magical, if there exist two total orders $<_{1},<_{2}$ on $V(G)$ such that $G_{<_{1},<_{2}}$ is magical. In this case, we say that the pair $\left(<_{1},<_{2}\right)$ witnesses $G$.

It easily follows from the above definition that if $G_{<_{1},<_{2}}$ is magical, then $G_{<_{1}}$ is a semi-comparability graph.

- Lemma 12. If $\mathcal{C}$ is a collection of grounded $x$-monotone curves, then the disjointness graph of $\mathcal{C}$ is magical.

Proof. Let $G$ be the disjointness graph of $\mathcal{C}$, and identify the vertices of $G$ with the elements of $\mathcal{C}$. For any $\gamma \in \mathcal{C}$, let $\left(0, y_{\gamma}\right)$ be the endpoint of $\gamma$ lying on the vertical axis $\{x=0\}$, and let $\left(x_{\gamma}, y_{\gamma}^{\prime}\right)$ be the other endpoint of $\gamma$.

Define the total orderings $<_{1}$ and $<_{2}$ on $V(G)$, as follows. Let $\gamma<_{1} \gamma^{\prime}$ if and only if $y_{\gamma}<y_{\gamma^{\prime}}$, and let $\gamma<_{2} \gamma^{\prime}$ if and only if $x_{\gamma}<x_{\gamma^{\prime}}$.

Suppose that for a triple $a, b, c \in \mathcal{C}$ we have that $a<_{1} b<_{1} c$ and $a b, b c \in E(G)$, but $a c \notin E(G)$. Then $a$ and $c$ intersect. Hence, $a, c$, and the ground curve $\{x=0\}$ enclose a region $A$, and $b \subset A$. This implies that the $x$-coordinate of the right endpoint of $b$ is smaller than the $x$-coordinates of the right endpoints of $a$ and $c$. Therefore, we have $b<_{2} a$ and $b<_{2} c$, showing that $G$ is magical.

- Lemma 13. Let $G$ be a magical graph. Then there exists a family $\mathcal{C}$ of grounded $x$-monotone curves such that the disjointness graph of $\mathcal{C}$ is isomorphic to $G$.

Proof. Let $n$ be the number of vertices of $G$. Let $<_{1}$ and $<_{2}$ be total orderings on $V(G)$ witnessing that $G$ is magical. For any vertex $v \in V(G)$, let $y(v) \in[n]$ denote the position of $v$ in the ordering $<_{1}$, and let $x(v)$ denote the position of $v$ in the ordering $<_{2}$.

For any $v \in V(G)$, we define an $x$-monotone curve $C_{v}$, which will be composed of $x(v)$ smaller $x$-monotone pieces, $C_{v}(1), \ldots, C_{v}(x(v))$, such that $C_{v}(i)$ starts at the point $(i-1, y(v))$, and ends at the point $(i, y(v))$. The pieces $C_{v}(i)$ are defined as follows.

Let $u_{i} \in V(G)$ be such that $x\left(u_{i}\right)=i$. If $u_{i}=v$ or there is an edge between $u_{i}$ and $v$, then let $C_{v}(i)$ be the horizontal line segment connecting $(i-1, y(v))$ and $(i, y(v))$. Otherwise, let $C_{v}(i)$ be the polygonal curve consisting of two segments whose three vertices are

$$
(i-1, y(v)), \quad\left(i-\frac{2}{3}, y\left(u_{i}\right)-\frac{1}{10}+\frac{y(v)}{10 n}\right),(i, y(v)) \quad \text { if } \quad y\left(u_{i}\right)<y(v)
$$

or

$$
(i-1, y(v)), \quad\left(i-\frac{1}{3}, y\left(u_{i}\right)+\frac{y(v)}{10 n}\right), \quad(i, y(v)) \quad \text { if } \quad y\left(u_{i}\right)>y(v) .
$$




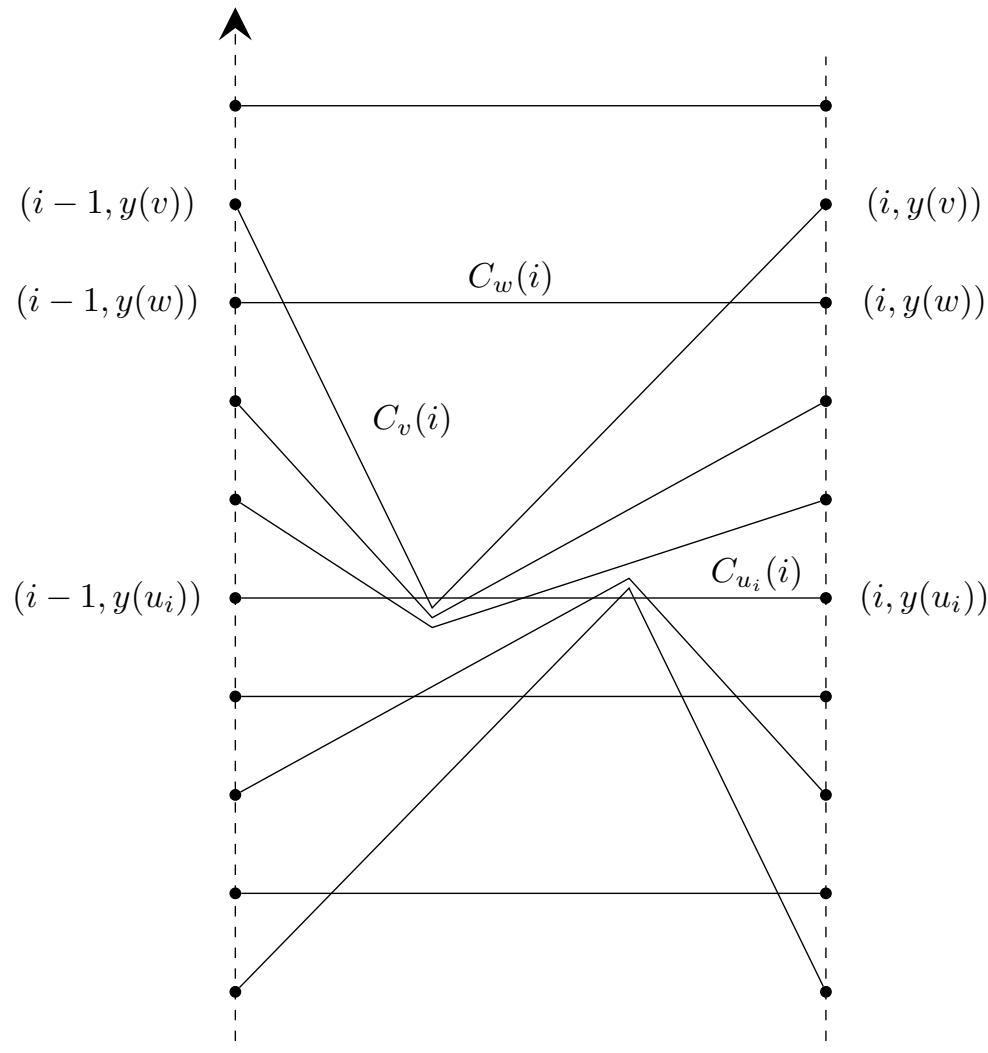

Figure 1 An illustration of the curves $C_{v}(i)$ in the proof of Lemma 13.

See Figure 1 for an illustration. One can easily check the following property of the curves $\left\{C_{v}(i)\right\}_{v \in V(G)}$. If $v, w \in V(G)$ are distinct vertices such that $C_{v}(i)$ and $C_{w}(i)$ intersect, then (i) $x(v), x(w) \geq i$.

(ii) Exactly one of $v$ and $w$ is joined to $u_{i}$ in $G$. Without loss of generality, assume that it is $w$.

(iii) Then $y\left(u_{i}\right) \leq y(w)<y(v)$ or $y(v)<y(w) \leq y\left(u_{i}\right)$.

Now we show that $G$ is the disjointness graph of $\mathcal{C}=\left\{C_{v}: v \in V(G)\right\}$.

If $v$ and $w$ are not joined by an edge in $G$, then $C_{v}(\min \{x(v), x(w)\})$ and $C_{w}(\min \{x(v)$, $x(w)\})$ intersect by definition, so $C_{v}$ and $C_{w}$ have a nonempty intersection.

Our task is reduced to showing that if $v$ and $w$ are joined by an edge, then $C_{v}$ and $C_{w}$ do not intersect. Suppose to the contrary that $C_{v}$ and $C_{w}$ intersect. Then there exists $i \in[\min \{x(v), x(w)\}-1]$ such that $C_{v}(i)$ and $C_{w}(i)$ intersect. Then either $y\left(u_{i}\right) \leq y(v), y(w)$ or $y\left(u_{i}\right) \geq y(v), y(w)$. Without loss of generality, let $y\left(u_{i}\right) \leq y(v), y(w)$, the other case can be handled in a similar manner. Again, without loss of generality, we can assume that $y(w)<y(v)$. Then $C_{v}(i)$ intersects $C_{u_{i}}(i)$, and $C_{w}(i)$ is disjoint from $C_{u_{i}}(i)$, or equivalently, $u_{i} w \in E(G)$, but $u_{i} v \notin E(G)$. However, this is impossible, because $w v \in E(G)$, so the triple $u_{i}, w, v$ would contradict the assumption that $G$ is magical.

By Lemma 13, in order to prove Theorem 2, it is enough to verify the corresponding statement for magical graphs. In other words, we have to prove the following.

Theorem 14. For every positive integer $k \geq 2$, there exists a magical graph $G$ such that $\omega(G)=k$ and $\chi(G)=\left(\begin{array}{c}k+1 \\ 2\end{array}\right)$. 


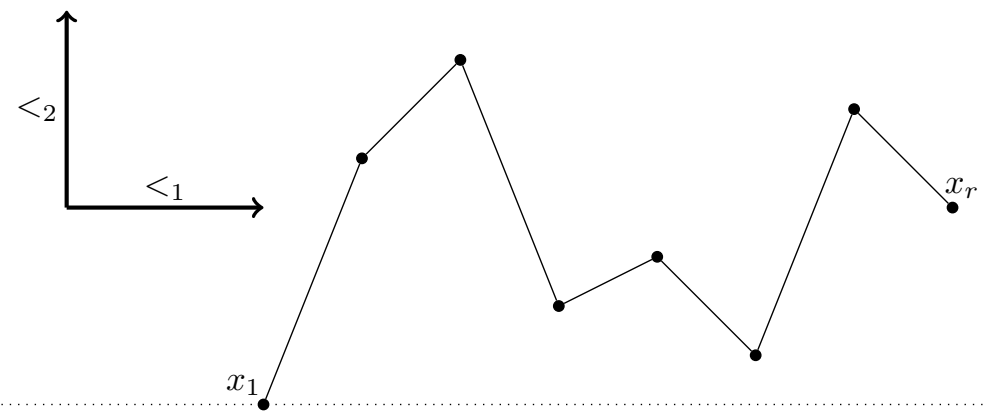

Figure 2 A mountain path. The dotted line shows the minimum of $x_{1}$ and $x_{r}$ in $<_{2}$, so all the other points of the path must be above it.

The rest of this section is devoted to the proof of this theorem. The proof is probabilistic and is inspired by a construction of Korándi and Tomon [18]. We shall consider a random double-ordered graph with certain parameters, and show that the smallest magical graph covering its edges meets the requirements in Theorem 14. To accomplish this plan, we first examine how the smallest magical graph covering the edges of a given double-ordered graph looks.

Let $G_{<_{1},<_{2}}$ be a double-ordered graph. A sequence of vertices $x_{1}, \ldots, x_{r} \in V(G)$ is said to form a mountain-path, if $x_{1}<_{1} \cdots<_{1} x_{r}, x_{i} x_{i+1} \in E(G)$ for every $i$, where $1 \leq i<r$, and either $x_{1}<_{2} x_{2}, \ldots, x_{r-1}$ or $x_{r}<_{2} x_{2}, \ldots, x_{r-1}$. See Figure 2 .

- Lemma 15. Let $G_{<_{1},<_{2}}$ be a double-ordered graph. There exists a unique minimal graph $G_{<_{1},<_{2}}^{\prime}$ on $V(G)$ such that $E(G) \subset E\left(G^{\prime}\right)$ and $G_{<_{1},<_{2}}^{\prime}$ is magical. Moreover, if $u, v \in V(G)$, then $u$ and $v$ are joined by an edge in $G^{\prime}$ if and only if there exists a mountain-path connecting $u$ and $v$.

Proof. Let $H=H_{<_{1},<_{2}}$ be any magical graph on the vertex set $V(G)$ such that $E(G) \subseteq E(H)$. Let $x_{1}, \ldots, x_{r}$ be a mountain-path in $G$ with $x_{1}<_{2} x_{r}$. Using the definition of magical graphs, it is easy to prove by induction on $i$ that $x_{1}$ and $x_{i}$ are joined by an edge in $E(H)$, for every $i>1$. Therefore, we have $x_{1} x_{r} \in E(H)$. (We can proceed similarly if $x_{r}<_{2} x_{1}$.)

With a slight abuse of notation, from now on let $H=H_{<_{1},<_{2}}$ denote the doubleordered graph on $V(G)$, in which $u$ and $v$ are joined by an edge if and only if there exists a mountain-path connecting $u$ to $v$. We will show that $H$ is magical, that is, for every triple $u, v, w \in V(G)$, the following holds: if $u<_{1} v<_{1} w$ such that $u v, v w \in E(H)$, and $u<_{2} v$ or $w<_{2} v$, then $u w \in E(H)$. As $u v, v w \in E(H)$, there exist two mountain-paths $u=x_{1}, x_{2}, \ldots, x_{r}=v$ and $v=x_{r}, x_{r+1}, \ldots, x_{s}=w$. By the assumption that $u<_{2} v$ or $w<_{2} v$, the path $u=x_{1}, \ldots, x_{s}=w$ is a mountain-path as well, so $u w \in E(H)$.

For the rest of the discussion, we need to introduce a few parameters that depend on $k$. Set $\lambda=1 / k^{2}, t=100 k^{2} \log k, h=3 t^{k^{2}} k^{2 k^{2}+8}, n=9 h$ and $p=t / n$.

Let $S=\left\{(a, b) \in[k]^{2}: a+b \geq k+1\right\}$. For each $(a, b) \in S$, let $A_{a, b}$ be a set of $n$ arbitrary points in the interior of the unit square $[a k+b, a k+b+1] \times[b k+a, b k+a+1]$ with distinct $x$ and $y$ coordinates, see Figure 3. Let $V=\bigcup_{(a, b) \in S} A_{a, b}$, and let $<_{1}$ and $<_{2}$ be the total orderings on $V$ induced by the $x$ and $y$ coordinates of the elements of $V$, respectively. A pair of vertices $\{u, v\}$ in $V$ is called available if $u \in A_{a, b}, v \in A_{a^{\prime}, b^{\prime}}$ with $(a, b) \neq\left(a^{\prime}, b^{\prime}\right)$.

Let $G_{0}$ denote the random graph on $V$ in which every available pair of vertices is connected by an edge with probability $p$, independently from each other. $G_{0}$ does not have any edge whose endpoints belong to the same set $A_{a, b}$. Let $G_{<_{1},<_{2}}^{\prime}$ be the minimal magical graph on $V$ containing all edges of $G_{0}$. 


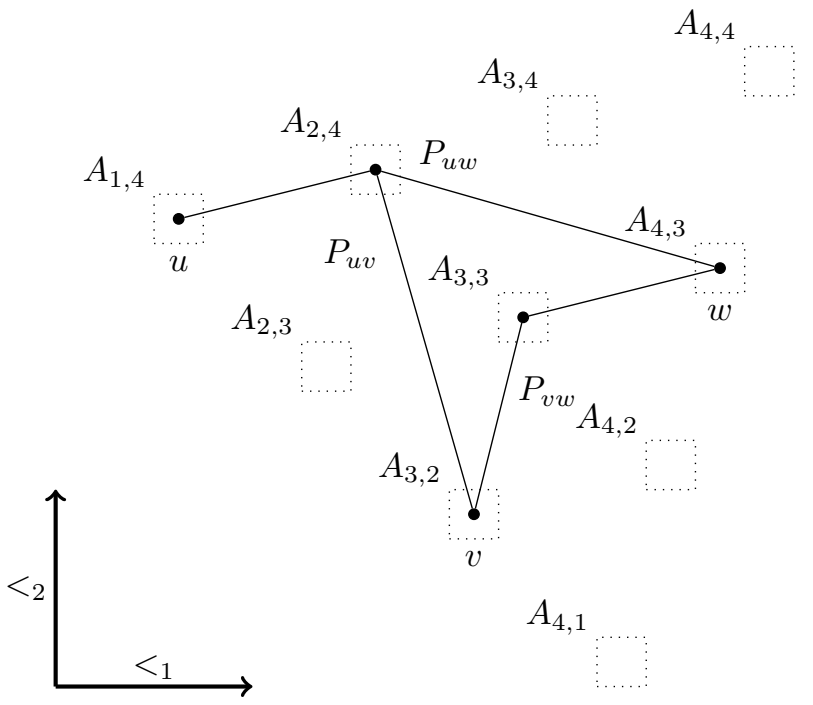

Figure 3 An illustration of the sets $A_{a, b}$ for $k=4$, and a hole $(u, v, w)$ which induces a triangle in $G^{\prime}$.

$\triangleright$ Claim 16. With probability at least $2 / 3, G^{\prime}$ has no independent set larger than $(1+\lambda) n$.

Proof. As $G_{0}$ is a subgraph of $G^{\prime}$, it is enough to show that $G_{0}$ has no independent set of size greater than $(1+\lambda) n$, with probability at least $2 / 3$.

Let $I \subset V$ such that $|I|>(1+\lambda) n$. Then there are at least $\lambda n^{2} / 2$ available pairs of vertices, whose both endpoints belong to $I$. Indeed, if $u \in A_{a, b}$, then $\{u, v\}$ is available for every $v \in\left(I \backslash A_{a, b}\right)$, so there are at least $\left|I \backslash A_{a, b}\right| \geq \lambda n$ available pairs containing $u$. Hence, the total number of available pairs in $I$ is at least $|I| \lambda n / 2>\lambda n^{2} / 2$.

Thus, the probability that $I$ is an independent set in $G_{0}$ is at most

$$
(1-p)^{\lambda n^{2} / 2}<e^{-p \lambda n^{2} / 2}=e^{-t \lambda n / 2} .
$$

As the number of $(1+\lambda) n$-sized subsets of $V$ is

$$
\left(\begin{array}{c}
|V| \\
(1+\lambda) n
\end{array}\right)<\left(\frac{e|V|}{(1+\lambda) n}\right)^{(1+\lambda) n}<\left(e k^{2}\right)^{(1+\lambda) n}
$$

the probability that there is a $(1+\lambda) n$-sized independent set is less than

$$
\left(e k^{2}\right)^{(1+\lambda) n} e^{-t \lambda n / 2}=e^{(1+2 \log k)(1+\lambda) n-t \lambda n / 2}<1 / 3 .
$$

A triple $(u, v, w) \in V^{3}$ is said to form a hole, if $u<_{1} v<_{1} w$ and $v<_{2} u, w$. Recall that $h=3 t^{k^{2}} k^{2 k^{2}+8}$.

$\triangleright$ Claim 17. Let $N$ be the number of holes in $V$ that induce a triangle in $G^{\prime}$. Then $\mathbb{E}(N)<h$.

Proof. Let $(u, v, w)$ be a hole, and let us bound the probability that $u, v, w$ induce a triangle in $G^{\prime}$. Suppose that $u \in A_{a_{1}, b_{1}}, v \in A_{a_{2}, b_{2}}$ and $w \in A_{a_{3}, b_{3}}$. We can assume that the pairs $\left(a_{1}, b_{1}\right),\left(a_{2}, b_{2}\right),\left(a_{3}, b_{3}\right)$ are distinct, otherwise $u, v, w$ cannot induce a triangle.

If $u v, v w, u w \in E\left(G^{\prime}\right)$, then there exist three mountain-paths, $P_{u, v}, P_{v, w}$ and $P_{u, w}$, with endpoints $\{u, v\},\{v, w\}$ and $\{u, w\}$, respectively. See Figure 3. Note that each of these paths intersects every $A_{a, b}$ in at most one vertex. As $u<_{1} v<_{1} w$, the only vertex in the intersection of $P_{u v}$ and $P_{v w}$ is $v$. Moreover, $P_{u w}$ cannot contain $v$ as $v<_{2} u$ and $v<_{2} w$. 
Consider the graph $P=P_{u v} \cup P_{v w} \cup P_{u w}$. It is a connected graph, but not a tree, because there are two distinct paths between $u$ and $w: P_{u v} \cup P_{v w}$ and $P_{u w}$. Hence, we have $|E(P)| \geq|V(P)|$. Let $\mathcal{P}$ denote the set of all such graphs $P$ that appear in $G_{0}$ with positive probability. Then

$\mathbb{P}\left(\{u, v, w\}\right.$ induces a triangle in $\left.G^{\prime}\right)=\mathbb{P}\left(P\right.$ is a subgraph of $G_{0}$ for some $\left.P \in \mathcal{P}\right)$

$$
\leq \sum_{P \in \mathcal{P}} \mathbb{P}\left(P \text { is a subgraph of } G_{0}\right) .
$$

For a fixed $P \in \mathcal{P}$, every edge of $P$ is present in $G_{0}$ independently with probability $p$. Hence, the probability that $P$ is a subgraph of $G_{0}$ is $p^{|E(P)|}$, which is at most $p^{|V(P)|}$. The number of graphs in $\mathcal{P}$ with exactly $m$ vertices is at most $\left(\begin{array}{c}|V| \\ m-3\end{array}\right)<\left(k^{2} n\right)^{m-3}$, as each member of $\mathcal{P}$ contains the vertices $u, v, w$. Finally, every member of $\mathcal{P}$ has at most $3|S| \leq 3 k^{2}$ vertices, so we can write

$$
\sum_{P \in \mathcal{P}} \mathbb{P}\left(P \text { is a subgraph of } G_{0}\right) \leq \sum_{m=3}^{3 k^{2}} p^{m}\left(k^{2} n\right)^{m-3}<3 t^{k^{2}} k^{2 k^{2}+2} n^{-3} .
$$

Since the number of holes in $V$ is at most $\left(\begin{array}{c}|V| \\ 3\end{array}\right)<|V|^{3}<k^{6} n^{3}$, we obtain

$$
\mathbb{E}(N)<3 t^{k^{2}} k^{2 k^{2}+8}=h .
$$

Applying Markov's inequality, the probability that $V$ contains more than $3 h$ holes that induce a triangle in $G^{\prime}$ is at most $1 / 3$. Hence, there exists a magical graph $G^{\prime}$ on $V$ such that $G^{\prime}$ has no independent set of size $(1+\lambda) n$, and $G^{\prime}$ contains at most $3 h$ triangles whose vertices form a hole. By deleting a vertex of each such hole in $G^{\prime}$, we obtain a magical graph $G$ with at least $|S| n-3 h$ vertices, which has no triangle whose vertices form a hole, and no independent set of size $(1+\lambda) n$.

First, we show that $\chi(G) \geq|S|=\left(\begin{array}{c}k+1 \\ 2\end{array}\right)$. Indeed, if $\chi(G) \leq|S|-1$, then $G$ contains an independent set of size

$$
\frac{|V(G)|}{|S|-1} \geq \frac{|S| n-3 h}{|S|-1}=\left(1+\frac{1}{|S|-1}\right) n-\frac{3 h}{|S|-1}>(1+\lambda) n,
$$

contradiction.

It remains to prove that $\omega(G)=k$. Clearly, $\omega(G) \geq k$, otherwise, by Lemma 8 , we would have $\chi(G) \leq\left(\begin{array}{l}k \\ 2\end{array}\right)$, contradicting the last paragraph. Thus, we have to show that $G$ has no clique of size $k+1$. For this, we need the following observation.

$\triangleright$ Claim 18. Let $K$ be a subset of $S$ that does not contain three points $\left(a_{1}, b_{1}\right),\left(a_{2}, b_{2}\right),\left(a_{3}, b_{3}\right)$ such that $a_{1}<a_{2} \leq a_{3}$ and $b_{2} \leq b_{1}$ and $b_{2}<b_{3}$. Then we have $|K| \leq k$.

Proof. We call $\left(a_{1}, b_{1}\right),\left(a_{2}, b_{2}\right),\left(a_{3}, b_{3}\right)$ a bad triple, if $a_{1}<a_{2} \leq a_{3}$ and $b_{2} \leq b_{1}$ and $b_{2}<b_{3}$.

Let $S=S_{k}$. We prove the claim by induction on $k$. For $k=1$, the claim is trivial. Suppose that $k \geq 2$ and that the statement has already been verified for $k-1$. We distinguish two cases.

Case 1: $K$ contains at most 1 element from the column $\{(k, b): b \in[k]\}$. Let

$$
K^{\prime}=\{(a, b):(a, b+1) \in K \text { and } a<k\} .
$$

Then $\left|K^{\prime}\right| \geq|K|-1$ and $K^{\prime} \subset S_{k-1}$ does not contain a bad triple. Thus, by the induction hypothesis, we have $\left|K^{\prime}\right| \leq k-1$, which implies that $|K| \leq k$. 
Case 2: $K$ contains 2 distinct elements of the form $(k, b)$ and $\left(k, b^{\prime}\right)$, where $b<b^{\prime}$. Then $K$ cannot contain $(a, k)$ for any $a \in[k-1]$, otherwise $(a, k),(k, b),\left(k, b^{\prime}\right)$ would be a bad triple. Thus, $K$ contains at most one element from the row $\{(a, k): a \in[k]\}$ (it might contain $(k, k))$. Let

$$
K^{\prime}=\{(a, b):(a+1, b) \in K \text { and } b \leq k-1\} .
$$

Again, $\left|K^{\prime}\right| \geq|K|-1$ and $K^{\prime} \subset S_{k-1}$ does not contain a bad triple. By the induction hypothesis, we have $\left|K^{\prime}\right| \leq k-1$ and, hence, $|K| \leq k$.

Now we are in a position to finish the proof of Theorem 14. Let $G$ denote the magical graph obtained from $G^{\prime}$ by deleting a vertex from each of its holes that form a triangle (see right before Claim 18). Suppose that $C \subset V$ is a clique in $G$. Then $C$ does not contain a hole and it intersects each $A_{a, b}$ in at most one vertex. Let $K=\left\{(a, b) \subset S: A_{a, b} \cap C \neq \emptyset\right\}$. The condition that $C$ does not contain a hole implies that $K$ does not contain three points $\left(a_{1}, b_{1}\right),\left(a_{2}, b_{2}\right),\left(a_{3}, b_{3}\right)$ such that $a_{1}<a_{2} \leq a_{3}$ and $b_{2} \leq b_{1}$ and $b_{2}<b_{3}$. Hence, by Claim 18, we have $|C|=|K| \leq k$. This completes the proof of Theorem 14 and, hence, the proof of Theorem 2 .

\section{Bounding function for curves that intersect a vertical line-Proof of Theorem 3}

A triple-ordered graph is a graph $G_{<_{1},<_{2},<_{3}}$ with three total orders $<_{1},<_{2},<_{3}$ on its vertex set.

- Definition 19. A triple-ordered graph $G_{<_{1},<_{2},<_{3}}$ is called double-magical, if there exist two magical graphs $G_{<_{1},<_{2}}^{1}$ and $G_{<_{1},<_{3}}^{2}$ on $V(G)$ such that $E\left(G_{<_{1},<_{2},<_{3}}\right)=E\left(G_{<_{1},<_{2}}^{1}\right) \cap$ $E\left(G_{<_{1},<_{3}}^{2}\right)$. An unordered graph $G$ is said to be double-magical, if there exist three total orders $<_{1},<_{2},<_{3}$ on $V(G)$ such that the triple-ordered graph $G_{<_{1},<_{2},<_{3}}$ is double-magical. We say that $G$ is witnessed by $\left(<_{1},<_{2},<_{3}\right)$.

By Lemmas 12 and 13, it is not hard to characterize disjointness graphs of $x$-monotone curves intersected by a vertical line.

- Lemma 20. Let $\mathcal{C}$ be a collection of $x$-monotone curves such that each member of $\mathcal{C}$ intersects the vertical line $l$. Then the disjointness graph of $\mathcal{C}$ is double-magical.

Proof. Without loss of generality, let $l=\{x=0\}$. For each $\gamma \in \mathcal{C}$, let $\left(-x_{\gamma}^{-}, y_{\gamma}^{-}\right)$be the left endpoint of $\gamma$, let $\left(0, y_{\gamma}\right)$ be the intersection point of $\gamma$ and $l$, and let $\left(x_{\gamma}^{+}, y_{\gamma}^{+}\right)$be the right endpoint of $\gamma$. Also, let $\gamma^{-}=\gamma \cap\{x \leq 0\}$ and $\gamma^{+}=\gamma \cap\{x \geq 0\}$, and let $\mathcal{C}^{-}=\left\{\gamma^{-}: \gamma \in \mathcal{C}\right\}$ and $\mathcal{C}^{+}=\left\{\gamma^{+}: \gamma \in \mathcal{C}\right\}$. Then $\mathcal{C}^{+}$is a collection of grounded curves, and $\mathcal{C}^{-}$is the reflection of a collection of grounded curves to the line $l$.

Let $G, G^{-}$and $G^{+}$be the disjointness graphs of $\mathcal{C}, \mathcal{C}^{-}$and $\mathcal{C}^{+}$, respectively, such that we identify $\gamma, \gamma^{-}$and $\gamma^{+}$as the vertices of these graphs for every $\gamma \in \mathcal{C}$. Then $E(G)=E\left(G^{-}\right) \cap E\left(G^{+}\right)$. Let $<_{1}$ be the total ordering on $\mathcal{C}$ defined by $\gamma<_{1} \gamma^{\prime}$ if $y_{\gamma}<y_{\gamma^{\prime}}$, let $<_{2}$ be the ordering defined by $\gamma<_{2} \gamma^{\prime}$ if $x_{\gamma}^{-}<x_{\gamma^{\prime}}^{-}$, and let $<_{3}$ be the ordering defined by $\gamma<_{3} \gamma^{\prime}$ if $x_{\gamma}^{+}<x_{\gamma^{\prime}}^{+}$. By Lemma $12, G_{<_{1},<_{2}}^{-}$and $G_{<_{1},<_{3}}^{+}$are magical, so $G_{<_{1},<_{2},<_{3}}$ is double-magical.

We can just as easily prove the converse of Lemma 20, using Lemma 13.

- Lemma 21. Let $G$ be a double-magical graph. Then there exists a collection of curves $\mathcal{C}$ such that each member of $\mathcal{C}$ has a nonempty intersection with the vertical line $\{x=0\}$, and the disjointness graph of $\mathcal{C}$ is isomorphic to $G$. 
Proof. Let $\left(<_{1},<_{2},<_{3}\right)$ be total orders on $V(G)$ witnessing that $G$ is double-magical, and let $G_{<_{1},<_{2}}^{1}, G_{<_{1},<_{3}}^{2}$ be two magical graphs on $V(G)$ such that $E(G)=E\left(G^{1}\right) \cap E\left(G^{2}\right)$.

Let $|V(G)|=n$. By Lemma 13, there exist $n$ grounded $x$-monotone curves $\gamma_{1}^{+}, \ldots, \gamma_{n}^{+}$ such that $\gamma_{i}^{+}$is contained in the nonnegative plane $\{x \geq 0\}$ with one endpoint at $(0, i)$, the disjointness graph of $\left\{\gamma_{1}^{+}, \ldots, \gamma_{n}^{+}\right\}$is $G^{1}$, and $\gamma_{i}^{+}$corresponds to the $i$-th vertex of $G^{1}$ in the order $<_{1}$. Also, there exist $n x$-monotone curves $\gamma_{1}^{-}, \ldots, \gamma_{n}^{-}$such that $\gamma_{i}^{-}$is contained in the nonpositive plane $\{x \leq 0\}$ with one endpoint at $(0, i)$, the disjointness graph of $\left\{\gamma_{1}^{-}, \ldots, \gamma_{n}^{-}\right\}$ is $G^{2}$, and $\gamma_{i}^{-}$corresponds to the $i$-th vertex of $G^{2}$ in the order $<_{1}$. For $i=1, \ldots, n$, set $\gamma_{i}=\gamma_{i}^{-} \cup \gamma_{i}^{+}$, then the disjointness graph of $\mathcal{C}=\left\{\gamma_{i}: i \in[n]\right\}$ is isomorphic to $G$, and every curve in $G$ has a nonempty intersection with the vertical line $\{x=0\}$.

For any double-magical graph $G=G_{<_{1},<_{2},<_{3}}$, define four partial orders $\prec_{1}, \prec_{2}, \prec_{3}, \prec_{4}$ on $V(G)$, as follows. For $a, b \in V(G)$, let

(i) $a \prec_{1} b \quad$ if $a<_{1} b, a<_{2} b, a<_{3} b$, and $a b \in E(G)$;

(ii) $a \prec_{2} b$ if $a<_{1} b, b<_{2} a, b<_{3} a$, and $a b \in E(G)$;

(iii) $a \prec_{3} b$ if $a<_{1} b, a<_{2} b, b<_{3} a$, and $a b \in E(G)$;

(iv) $a \prec_{4} b$ if $a<_{1} b, b<_{2} a, a<_{3} b$, and $a b \in E(G)$.

It follows easily from the definition of double-magical graphs that these are indeed partial orders. Moreover, they satisfy the following conditions.

(1) If $a b \in E(G)$, then $a$ and $b$ are comparable by precisely one of these 4 partial orders.

(2) For any $a, b, c \in V(G)$ and $i \in[4]$, if $a \prec_{1} b$ and $b \prec_{i} c$, then $a c \in E(G)$.

(3) For any $a, b, c \in V(G)$ and $i \in[4]$, if $a \prec_{i} b$ and $b \prec_{2} c$, then $a c \in E(G)$.

- Theorem 22. Let $G$ be a double-magical graph. If $\omega(G)=k$, then $\chi(G) \leq \frac{k+1}{2}\left(\begin{array}{c}k+2 \\ 3\end{array}\right)$.

Proof. Let $<_{1},<_{2},<_{3}$ be total orders on $V(G)$ witnessing $G$, and let $\prec_{1}, \prec_{2}, \prec_{3}, \prec_{4}$ denote the partial orders defined above. Clearly, there is no chain of length $k+1$ with respect to any of the partial orders $\prec_{i}$, because that would contradict the assumption $\omega(G)=k$.

For $h=1, \ldots, k$, let $S_{h}$ denote the set of vertices $v \in V(G)$ for which the size of a longest $\prec_{1}$-chain with maximal element $v$ is $k-h+1$. Then the sets $S_{1}, \ldots, S_{k}$ form a partition of $V(G)$, where each $S_{h}$ is a $\prec_{1}$-antichain that contains no clique of size $h+1$. Indeed, suppose that $C \subset S_{h}$ induces a clique of size $h+1$ in $G$, and consider the smallest vertex $v \in C$ with respect to the order $<_{1}$. There exists a $\prec_{1}$-chain $D$ of size $k-h+1$ ending at $v$. This implies that for every $a \in D$ and $b \in C$, we have $a \prec_{1} v$ and $v \prec_{i} b$ for some $i \in\{2,3,4\}$. Then, by (2), we would have $a b \in E(G)$. Hence, $D \cup C$ would induce a clique of size $k+1$, contradiction.

For $h=1, \ldots, k$ and $m=1, \ldots, h$, let $S_{h, m}$ denote the set of vertices in $S_{h}$ for which the largest $\prec_{2}$-chain in $S_{h}$ with smallest element $v$ has size $h-m+1$. As $\omega\left(G\left[S_{h}\right]\right) \leq h$, the sets $S_{h, 1}, \ldots, S_{h, h}$ are $\prec_{1^{-}}$and $\prec_{2}$-antichains partitioning $S_{l}$. Further, $S_{h, m}$ contains no clique of size $m+1$. Otherwise, if $C \subset S_{h, m}$ forms a clique of size $m+1$ in $G$, then consider the largest vertex $v \in C$ with respect to the order $<_{1}$. There exists a $\prec_{2}$-chain $D$ of size $h-m+1$ whose smallest element is $v$. Hence, for every $a \in C$ and $b \in D$, we have $a \prec_{i} v$ and $v \prec_{2} b$ for some $i \in\{3,4\}$, which implies, by (3), that $a b \in E(G)$. Hence, $C \cup D$ would induce a clique of size $h+1$ in $S_{h}$, contradiction.

Thus, we obtained that $S_{h, m}$ is a $\prec_{1}$ - and $\prec_{2}$-antichain, which does not contain a clique of size $m+1$. In particular, the size of the longest $\prec_{3^{-}}$and $\prec_{4}$-chains in $S_{l, m}$ is at most $m$. This means that $G\left[S_{h, m}\right]$ can be properly colored with $m^{2}$ colors. Indeed, set the color of $v \in S_{h, m}$ to be $\phi(v)=(r, q)$, where $r$ is the size of the largest $\prec_{3}$-chain with smallest element $v$, and $q$ is the size of the largest $\prec_{4}$-chain with smallest element $v$. Then $\phi: S_{h, m} \rightarrow[m]^{2}$ is a proper coloring of $G\left[S_{h, m}\right]$. 
As $S_{h}=\bigcup_{m=1}^{h} S_{h, m}$, we have

$$
\chi\left(G\left[S_{h}\right]\right) \leq \sum_{m=1}^{h} \chi\left(G\left[S_{h, m}\right]\right) \leq \sum_{m=1}^{h} m^{2}=\frac{h(h+1)(2 h+1)}{6} .
$$

Finally, since $V(G)=\bigcup_{h=1}^{k} S_{h}$, we obtain

$$
\chi(G) \leq \sum_{h=1}^{k} \chi\left(G\left[S_{h}\right]\right) \leq \sum_{h=1}^{k} \frac{h(h+1)(2 h+1)}{6}=\frac{k+1}{2}\left(\begin{array}{c}
k+2 \\
3
\end{array}\right) .
$$

\section{Construction of double-magical graphs-Proof of Theorem 4}

In view of 21 , to prove Theorem 4 , it is enough to construct a double-magical graph with the desired clique and chromatic numbers.

- Theorem 23. For every positive integer $k \geq 2$, there exists a double-magical graph $G$ satisfying $\omega(G)=k$ and $\chi(G)=\frac{k+1}{2}\left(\begin{array}{c}k+2 \\ 3\end{array}\right)$.

In the rest of this section, we prove this theorem. The proof of Lemma 22 reveals a lot about the structure of double-magical graphs satisfying the properties of Theorem 23, if they exist. To construct them, we use reverse engineering.

For any vector $\mathbf{v} \in \mathbb{R}^{d}$ and any $j \in[d]$, let $\mathbf{v}(j)$ denote the $j$ th coordinate of $\mathbf{v}$. The sign vector of $\mathbf{v} \in \mathbb{R}^{d}$ is the $d$-dimensional vector $\operatorname{sg}(\mathbf{v})$ with

$$
\operatorname{sg}(\mathbf{v})(i)= \begin{cases}1 & \text { if } \mathbf{v}(i)>0 \\ -1 & \text { if } \mathbf{v}(i)<0 \\ 0 & \text { if } \mathbf{v}(i)=0\end{cases}
$$

Let $\mathbf{v}_{1}=(1,1,1), \mathbf{v}_{2}=(1,-1,-1), \mathbf{v}_{3}=(1,1,-1)$ and $\mathbf{v}_{4}=(1,-1,1)$. For any $\mathbf{i} \in[k]^{4}$, let

$$
P(\mathbf{i})=k^{3} \mathbf{i}(1) \mathbf{v}_{1}+k^{2} \mathbf{i}(2) \mathbf{v}_{2}+k \mathbf{i}(3) \mathbf{v}_{3}+\mathbf{i}(4) \mathbf{v}_{4} .
$$

These $k^{4}$ points have the useful property that if $\mathbf{i} \neq \mathbf{i}^{\prime}$, then the relative position of $P(\mathbf{i})$ and $P\left(\mathbf{i}^{\prime}\right)$ depends only on the smallest coordinate in which $\mathbf{i}$ and $\mathbf{i}^{\prime}$ differ. We refer to this property as the LEX property (short for "lexicographic"), which is formally defined as follows.

LEX property. Let $\mathbf{i}, \mathbf{i}^{\prime} \in[k]^{4}$ be such that $\mathbf{i} \neq \mathbf{i}^{\prime}$, and let $r$ be the smallest index such that $\mathbf{i}(r) \neq \mathbf{i}^{\prime}(r)$. If $\mathbf{i}(r)>\mathbf{i}^{\prime}(r)$, then

$$
\operatorname{sg}\left(P(\mathbf{i})-P\left(\mathbf{i}^{\prime}\right)\right)=\mathbf{v}_{r} .
$$

Let

$$
S=\left\{\mathbf{i} \in[k]^{4}: \mathbf{i}(1)+\mathbf{i}(2) \leq k+1, \mathbf{i}(2) \geq \mathbf{i}(3), \text { and } \mathbf{i}(2) \geq \mathbf{i}(4)\right\},
$$

so that we have

$$
|S|=\sum_{i=1}^{k}(k+1-i) i^{2}=\frac{k+1}{2}\left(\begin{array}{c}
k+2 \\
3
\end{array}\right) .
$$

An ordered triple of points $(u, v, w) \in \mathbb{R}^{3} \times \mathbb{R}^{3} \times \mathbb{R}^{3}$ is called a hole if $u(1)<v(1)<w(1)$, and either $v(2)<\min \{u(2), w(2)\}$, or $v(3)<\min \{u(3), w(3)\}$. 


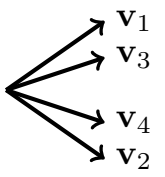

$P(3,1,1,1)$

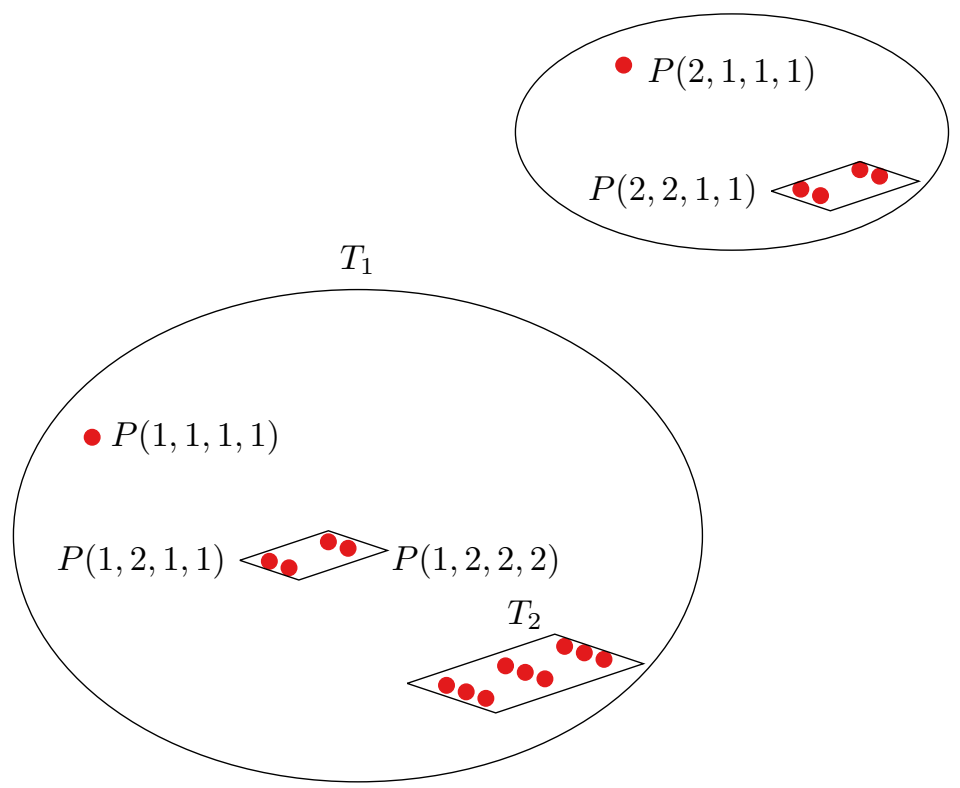

Figure 4 An illustration of the points $P(\mathbf{i})$ for $\mathbf{i} \in S, k=3$.

$\triangleright$ Claim 24. Let $H \subset S$. If the set $\{P(\mathbf{i}): \mathbf{i} \in H\}$ does not contain a hole, then $|H| \leq k$.

Proof. Let $S=S_{k}$. We prove this claim by induction on $k$. If $k=1, S$ contains one element, so there is nothing to prove.

Suppose that $k \geq 2$. Let $T_{1}=\{\mathbf{i} \in S: \mathbf{i}(1)=1\}$ and $H_{1}=H \cap T_{1}$. (See Figure 4 for an illustration.) We distinguish two cases.

Case 1: $\left|H_{1}\right| \leq 1$. Define

$$
H^{\prime}=\left\{\left(i_{1}-1, i_{2}, i_{3}, i_{4}\right):\left(i_{1}, i_{2}, i_{3}, i_{4}\right) \in H \backslash H_{1}\right\} .
$$

Then $H^{\prime} \subset S_{k-1}$ and $H^{\prime}$ does not contain a hole. Hence, we obtain $\left|H^{\prime}\right| \leq k-1$, by the induction hypothesis. On the other hand, $\left|H^{\prime}\right| \geq|H|-1$, which yields that $|H| \leq k$.

Case 2: $\left|H_{1}\right| \geq 2$. In this case, we must have $H=H_{1}$. Otherwise, choose $\mathbf{i}, \mathbf{i}^{\prime} \in H_{1}$, $\mathbf{j} \in H \backslash H_{1}$, and let $u=P(\mathbf{i}), v=P\left(\mathbf{i}^{\prime}\right)$, and $w=P(\mathbf{j})$. Assume without loss of generality that $u(1)<v(1)$. Then $u(1)<v(1)<w(1)$, and by the LEX property we have $w(2)>\max \{u(2), v(2)\}$ and $w(3)>\max \{u(3), v(3)\}$. Therefore, if $(u, v, w)$ is not a hole, then we must have $u(2)<v(2)<w(2)$ and $u(3)<v(3)<w(3)$. However, this means that $\operatorname{sg}(v-u)=(1,1,1)=\mathbf{v}_{1}$, which contradicts the LEX property, as $\mathbf{i}(1)=\mathbf{i}^{\prime}(1)$.

Hence, we can assume that $H=H_{1} \subset T_{1}$. Let $T_{2}=\{\mathbf{i} \in S: \mathbf{i}(1)=1, \mathbf{i}(2)=k\} \subset T_{1}$ and $H_{2}=H \cap T_{2}$. Again, we distinguish two subcases. 
Subcase 1: $\left|H_{2}\right| \leq 1$. Define $H^{\prime}=H \backslash H_{2}$. Then $H^{\prime} \subset S_{k-1}$ and $H^{\prime}$ does not contain a hole, which yields, by the induction hypothesis, that $\left|H^{\prime}\right| \leq k-1$. On the other hand, $\left|H^{\prime}\right| \geq|H|-1$, so $|H| \leq k$.

Subcase 2: $\left|H_{2}\right| \geq 2$. In this case, we show that $H=H_{2}$. Otherwise, let $\mathbf{i}, \mathbf{i}^{\prime} \in H_{2}$, $\mathbf{j} \in H \backslash H_{2}$, and $u=P(\mathbf{j}), v=P(\mathbf{i})$ and $w=P\left(\mathbf{i}^{\prime}\right)$. Assume without loss of generality that $v(1)<w(1)$. Then $u(1)<v(1)<w(1), u(2)>\max \{v(2), w(2)\}$, and $u(3)>\max \{v(3), w(3)\}$, by the LEX property. Thus, $(u, v, w)$ is a hole, unless $u(2)>v(2)>w(2)$ and $u(3)>v(3)>w(3)$, which would mean that the $\operatorname{sg}(w-v)=$ $(1,-1,-1)=\mathbf{v}_{2}$. However, this contradicts the LEX property, because $\mathbf{i}(2)=\mathbf{i}^{\prime}(2)$.

Hence, we can suppose that $H=H_{2} \subset T_{2}$. Here, $T_{2}$ is partitioned into $k$ sets $U_{1}, \ldots, U_{k}$, where $U_{l}=\{(1, k, l, m): m=1, \ldots, k\}$ for $l=1, \ldots, k$. Note that $\left|U_{l}\right|=k$. We show that $H$ is either completely contained in one of the sets $U_{l}$, or $H$ intersects each of $U_{1}, \ldots, U_{k}$ in at most one element. In either case, we get $|H| \leq k$. Suppose to the contrary that there exists $l \neq l^{\prime}$ and three elements $\mathbf{i}, \mathbf{i}^{\prime} \in U_{l} \cap H, \mathbf{j} \in U_{l^{\prime}} \cap H$. Let $u=P(\mathbf{i}), v=P\left(\mathbf{i}^{\prime}\right)$, and $w=P(\mathbf{j})$. Without loss of generality, assume that $u(1)<v(1)$. Now there are two cases depending on the order of $l$ and $l^{\prime}$. If $l<l^{\prime}$, then by the LEX property $u(1)<v(1)<w(1), v(2)<u(2)<w(2)$, and $w(3)<u(3)<v(3)$, so $(u, v, w)$ is a hole. If $l^{\prime}<l$, then $w(1)<u(1)<v(1), w(2)<v(2)<u(2)$, and $u(3)<v(3)<w(3)$, so $(w, u, v)$ is a hole.

The rest of the proof of Theorem 23 is very similar to that of the proof of Theorem 14 . We omit its proof here.

\section{Concluding remarks}

We proved that the best $\chi$-bounding function for the family of disjointness graphs of $x$ monotone curves satisfies $f(k)=\Theta\left(k^{4}\right)$. What can we say about the chromatic number of $K_{k}$-free families of disjointness graphs of segments or convex sets? For both of these families, the best known upper bound on the chromatic number is also $O\left(k^{4}\right)$, but there are no matching lower bounds.

\section{References}

1 Pankaj K Agarwal and Nabil H Mustafa. Independent set of intersection graphs of convex objects in 2D. Computational Geometry, 34(2):83-95, 2006.

2 Edgar Asplund and Branko Grünbaum. On a coloring problem. Mathematica Scandinavica, 8(1):181-188, 1960.

3 Peter Brass, William OJ Moser, and János Pach. Research problems in discrete geometry. Springer Science \& Business Media, 2006.

4 James Perkins Burling. On Coloring Problems of Families of Polytopes, 1965.

5 Sergio Cabello, Jean Cardinal, and Stefan Langerman. The clique problem in ray intersection graphs. Discrete \&3 computational geometry, 50(3):771-783, 2013.

6 Márcia R Cerioli, Luerbio Faria, Talita O Ferreira, and Fábio Protti. On minimum clique partition and maximum independent set on unit disk graphs and penny graphs: complexity and approximation. Electronic Notes in Discrete Mathematics, 18:73-79, 2004.

7 Robert P Dilworth. A decomposition theorem for partially ordered sets. Annals of mathematics, pages 161-166, 1950.

8 Adrian Dumitrescu and János Pach. Minimum clique partition in unit disk graphs. In Graphs and Combinatorics, volume 27(3), pages 399-411. Springer, 2011. 
9 Stephan Eidenbenz and Christoph Stamm. Maximum clique and minimum clique partition in visibility graphs. In IFIP International Conference on Theoretical Computer Science, pages 200-212. Springer, 2000.

10 Jacob Fox and János Pach. Computing the independence number of intersection graphs. In Proceedings of the twenty-second annual ACM-SIAM symposium on Discrete algorithms, pages 1161-1165. Society for Industrial and Applied Mathematics, 2011.

11 Zoltán Füredi. The maximum number of unit distances in a convex n-gon. J. Comb. Theory, Ser. A, 55(2):316-320, 1990.

12 Michael R Garey and David S Johnson. Computers and intractability, volume 29. wh freeman New York, 2002.

13 Fanika Gavril. Algorithms for a maximum clique and a maximum independent set of a circle graph. Networks, 3(3):261-273, 1973.

14 András Gyárfás. On the chromatic number of multiple interval graphs and overlap graphs. Discrete mathematics, 55(2):161-166, 1985.

15 András Gyárfás. Problems from the world surrounding perfect graphs. Applicationes Mathematicae, 19(3-4):413-441, 1987.

16 J Mark Keil and Lorna Stewart. Approximating the minimum clique cover and other hard problems in subtree filament graphs. Discrete Applied Mathematics, 154(14):1983-1995, 2006.

17 Chaya Keller, Shakhar Smorodinsky, and Gábor Tardos. On Max-Clique for intersection graphs of sets and the Hadwiger-Debrunner numbers. In Proceedings of the Twenty-Eighth Annual ACM-SIAM Symposium on Discrete Algorithms, pages 2254-2263. SIAM, 2017.

18 Dániel Korándi and István Tomon. Improved Ramsey-type results in comparability graphs. arXiv preprint, 2018. arXiv:1810.00588.

19 Alexandr Kostochka. Coloring intersection graphs of geometric figures with a given clique number. Contemporary mathematics, 342:127-138, 2004.

20 Alexandr Kostochka and Jan Kratochvíl. Covering and coloring polygon-circle graphs. Discrete Mathematics, 163(1-3):299-305, 1997.

21 Alexandr Kostochka and Jaroslav Nešetril. Chromatic number of geometric intersection graphs. In 1995 Prague Midsummer Combinatorial Workshop, volume 95(309), pages 43-45. KAM Series, 1995.

22 Alexandr V Kostochka. Upper bounds on the chromatic number of graphs. Trudy Inst. Mat, 10:204-226, 1988.

23 Jan Kratochvíl and Jirí Matousek. Intersection graphs of segments. Journal of Combinatorial Theory, Series B, 62(2):289-315, 1994.

24 Jan Kratochvíl and Jaroslav Nešetřil. Independent set and clique problems in intersectiondefined classes of graphs. Commentationes Mathematicae Universitatis Carolinae, 31(1):85-93, 1990.

25 Jan Kynčl. Ramsey-type constructions for arrangements of segments. European Journal of Combinatorics, 33(3):336-339, 2012.

26 David Larman, Jiří Matoušek, János Pach, and Jenő Törőcsik. A Ramsey-Type Result for Convex Sets. Bulletin of the London Mathematical Society, 26(2):132-136, 1994.

27 Michał Lasoń, Piotr Micek, Arkadiusz Pawlik, and Bartosz Walczak. Coloring intersection graphs of arc-connected sets in the plane. Discrete \& Computational Geometry, 52(2):399-415, 2014.

28 Sean McGuinness. Colouring arcwise connected sets in the plane I. Graphs and Combinatorics, 16(4):429-439, 2000.

29 Leon Mirsky. A dual of Dilworth's decomposition theorem. The American Mathematical Monthly, 78(8):876-877, 1971.

30 Torsten Mütze, Bartosz Walczak, and Veit Wiechert. Realization of shift graphs as disjointness graphs of 1-intersecting curves in the plane. arXiv preprint, 2018. arXiv:1802.09969.

31 János Pach and Gábor Tardos. Forbidden paths and cycles in ordered graphs and matrices. Israel Journal of Mathematics, 155(1):359-380, 2006. 
32 János Pach, Gábor Tardos, and Géza Tóth. Disjointness graphs of segments. arXiv preprint, 2017. arXiv:1704.01892.

33 János Pach and Jenő Töröcsik. Some geometric applications of Dilworth's theorem. Discrete 8 Computational Geometry, 12(1):1-7, 1994.

34 Arkadiusz Pawlik, Jakub Kozik, Tomasz Krawczyk, Michał Lasoń, Piotr Micek, William T Trotter, and Bartosz Walczak. Triangle-free intersection graphs of line segments with large chromatic number. Journal of Combinatorial Theory, Series B, 105:6-10, 2014.

35 Alexandre Rok and Bartosz Walczak. Outerstring graphs are $\chi$-bounded. In Symposium on Computational Geometry, page 136, 2014.

36 Alexandre Rok and Bartosz Walczak. Coloring curves that cross a fixed curve. Discrete \& Computational Geometry, pages 1-22, 2017.

37 Andrew Suk. Coloring intersection graphs of x-monotone curves in the plane. Combinatorica, 34(4):487-505, 2014.

38 Kenneth Jay Supowit. TOPICS IN COMPUTATIONAL GEOMETRY. UIUCDCS-R, 1982. 